Document downloaded from:

http://hdl.handle.net/10251/112509

This paper must be cited as:

Sen, AK.; Botet, R.; Vilaplana Cerda, RI.; Choudhury, NR.; Gupta, R. (2017). The effect of porosity of dust particles on polarization and color with special reference to comets. Journal of Quantitative Spectroscopy and Radiative Transfer. 198:164-178.

doi:10.1016/j.jqsrt.2017.05.009

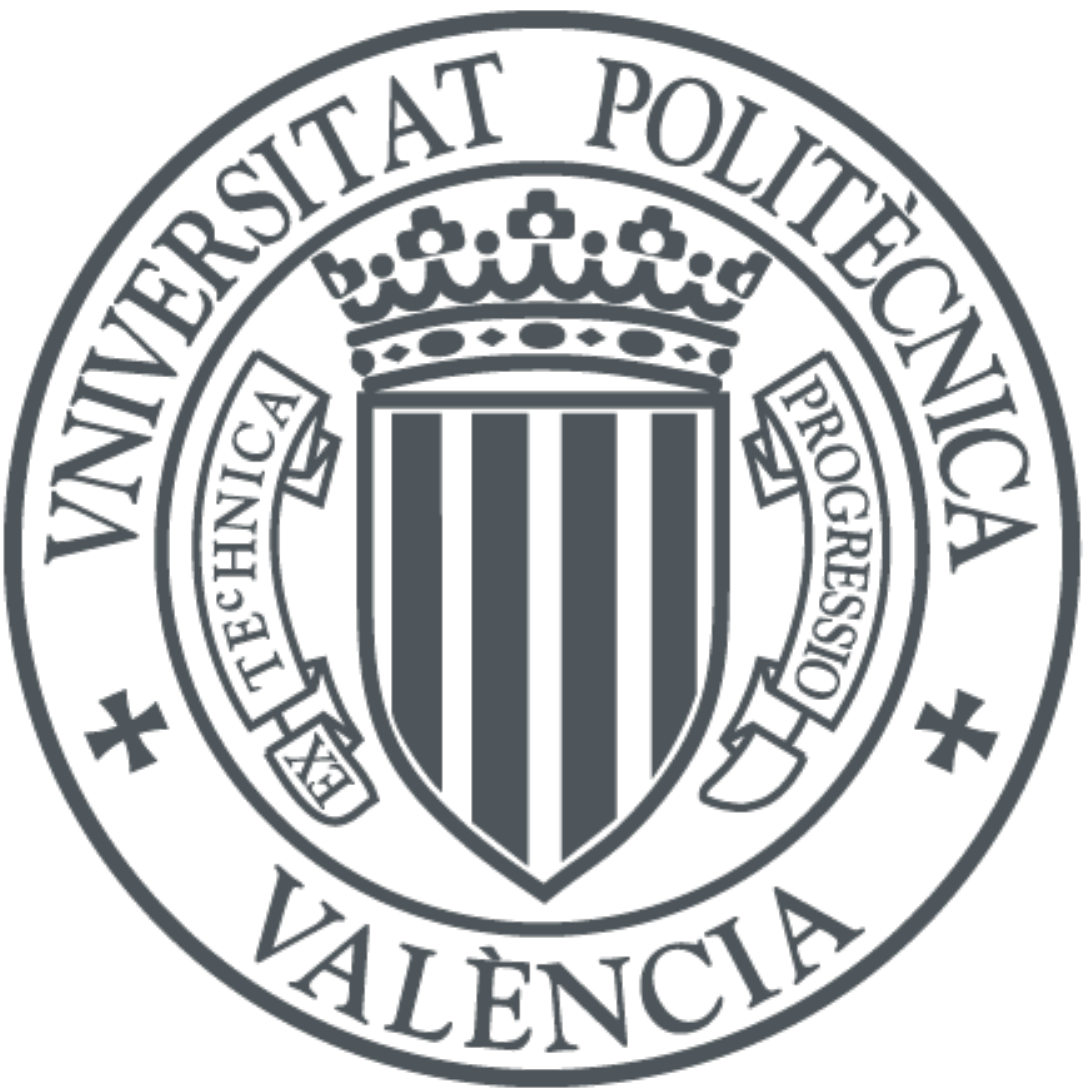

The final publication is available at

http://doi.org/10.1016/j.jqsrt.2017.05.009

Copyright Elsevier

Additional Information 


\title{
The effect of porosity of dust particles on polarization and color with special reference to comets
}

\author{
A. K. Sen \\ Department of Physics, Assam University, Silchar-788011, India \\ asokesen@yahoo.com \\ $R$ Botet
}

Laboratoire de Physique des Solides UMR8502, Université Paris-Sud, Université

Paris-Saclay, Orsay 91405, France

robert.botet@u-psud.fr

R. Vilaplana

Dpto. de Física Aplicada, Universidad Politécnica de Valencia, E.P.S.A. Alcoy 03801, Spain

rovilap@fis.upv.es

Naznin R Choudhury

Department of Physics, Assam University, Silchar-788011, India

nazninrahimchoudhury@gmail.com

Ranjan Gupta

IUCAA, Ganeshkhinde, Pune 411007, India

rag@iucaa.in

\begin{abstract}
Cosmic dust particles are mostly responsible for polarization of the light that we observe from astrophysical objects. They also lead to color-extinction, thermal re-emission and other scattering related phenomena. Micrometric dust particles are often made of smaller constituent (nanometric grains). They are characterized by their size (average radius), chemical composition and morphology (including porosity). In the present work, we address the question of the role of the dust particle porosity on light polarization and color, using Discrete Dipole Approximation (DDA) light scattering code. To this purpose, we develop an algorithm to generate dust particles of arbitrary values of porosity. In brief, starting from a compact spherical ensemble of dipoles,randomly the dipoles are removed one by one, such that the remaining dipoles remain connected within their neighbours. We stop the removal process when the desired porosity is obtained. Then we compute and study the optical properties of the porous dust particle.

The main objective of this paper is to develop a tool to generate dust particles with an arbitrary value of porosity and to study the effect of porosity on their light scattering properties. As a possible application, we simulate cometary polarization and color values which grossly match with the observed ones for the comet 1P/Halley, leaving scope for future work.
\end{abstract}

Keywords: dust: extinction- dust: polarization- dust: color- comet:general- porousaggregate 


\section{Introduction}

Ever since the first polarization measurements have been made for astronomical objects, scientists are using polarimetry as a tool to probe astrophysical processes in celestial environments. It has been the case for the comets since the first observation of the great comet C/1819 N1 tail polarization by Arago (von Humboldt 1997) and his conclusions that comet was not a self-luminous object and that its polarization was the result of reflection of the sunlight on material lost by the comet. It has also been the case for the interstellar dust particles, evidenced independently by Hall (1949) and Hiltner (1949) from their measurements of stars polarization that was interpreted as starlight being differentially absorbed ( for the two orthogonal components of electric vectors) by elongated small dichroic dust particles aligned by the magnetic field (Greenberg \& Hage 1990).

Nowadays, among the various processes that may cause polarization from remote astronomical sources, light scattering by small dust particles is expected to be the most common case. Such cosmic dust particles may appear naturally under the form either of dispersed particles (comet tails, reflection nebula, etc.) or of deposited particles (regoliths, pond-like deposits, etc.). Information about shape and composition of the cosmic dust particles should come from theory, which tells us how the light polarization is related to the various dust particle properties and to the scattering geometries.

Theory of light polarization from dust particles should start from the quantitative explanation of sky polarization by Rayleigh (1871). Indeed, at a time when the concept of molecules was unclear (Maxwell 1873), Rayleigh studied the light scattered by minute dust particles ("all small compared with any of the wave-lengths" (Rayleigh 1871) without reference to the nature of the atmospheric molecules. Thus, the Rayleigh bipolar scattering theory applies fully to any particle much smaller than the wavelength.

Later, Mie proposed in 1908 the analytical solution of the scattering of a plane electromagnetic wave by a perfect homogeneous compact dielectric sphere of any radius. This solution (van de Hulst 1957) provided a real breakthrough in the field, since it allowed for the first time to tackle the relation between polarization from dust particles and the dust particle sizes (in all the following, "dust particle size" means the typical radius of the dust particle, thus we consider dust particles which are not essentially anisotropic).

Generally, dust particles are not expected to be perfect spheres, then the Mie theory is only approximation of the real process - though a very important one indeed. The advent of the computer in the 1970s has allowed to deal with light scattered by other shapes and structures. Over the years, numerical tools were made available to compute light scattered by irregularly shaped dust particles, which were expected to be most naturally occurring in astrophysical conditions. Such numerical simulation codes include T-matrix (Mishchenko et al. 1994), DDSCAT (Draine 2000), FDTD (Taflove \& Hagness 1995), to mention just a few.

Over the years, as we have understood that astrophysical dust particles should be made of disordered porous / fluffy dust particles, scientists came up with various nu- 
merical codes to simulate such dust particles. That way, algorithms were developed to simulate formation processes of irregularly shaped dust particles under realistic physical conditions. The most popular code, the Cluster-Cluster Aggregation mode (Jullien $\&$ Botet 1987) and its variants, provide reliable numerical analogues for astrophysical dust particles made of aggregates of small grains. Such aggregates are often fractal hence of fluffy structures, though they may exhibit irregular compact shapes as well, depending on the conditions.

There is a set of commonly accepted terminologies that is worth recalling here, since it may depend on the domain of physics in which dust particles are considered. In the context of astrophysics, a "grain" is a tiny solid particle much smaller than a characteristic length scale (in the present context: much smaller than the wavelength , that is nanometric in size). A grain is generally assumed to be spherical in shape because the surface irregularities of such a minute solid are irrelevant for the wavelengths considered. An absence of a grain in a compact dust particle will introduce porosity. We use the words fluffy or porous with the same intention to mean voids in the aggregates.

Finally, a grain can be represented by a collection of dipoles to comply with the Discrete-Dipole Approximation (DDA), which is the base of the DDSCAT calculations (Draine 2000). The dipoles are the electromagnetic counterparts of nanometric compact domains of standard shape, occupying a definite position in a three dimensional regular lattice structure and having complex refractive index assigned to each individual dipole (Shen et al. 2008, 2009).

Porous / fluffy aggregates are expected to exist everywhere in outer space, from our solar system to the interstellar medium and also in other galaxies. However, in this paper the results that we obtain from our simulations will be compared with the data from comets. This is primarily due to two reasons: (1) unlike other celestial objects, for comets we get light scattering data over widest possible scattering angle range ( hence puts more constraints on any model) and (2) for some comets like 1P/Halley, a very large data base is available to match observed polarization and intensities, with the simulated values. However, our goal here is not to match perfectly an observational database, but to check that our model is suitable to approach several light scattering properties of a well-doumented comet.

Indeed, there is huge amount of data for comets observed at various scattering geometries, and the ground-based data are complemented by in situ space measurements.

Brownlee (1985) directly detected for the first time the porous/fluffy dust particles in our upper atmosphere through in situ measurements. In that work it was stated that, the collected IDP (Interplanetary Dust Particles) have densities ranging from 0.7 to 2.0 $\mathrm{g}$ per $\mathrm{cc}$ for sizes of few micrometers. The BCCA or BPCA structures with Silicate compositions are inconsistent with such bulk densities, though there have been recent works where such structures were tentatively used to explain cometary polarization ( Das et al. 2011 and references therein). In those works, it can be noted that neither BCCA nor BPCA could solely fit the observational data on comets. The bulk density of dust particles in comet $81 \mathrm{P} /$ Wild2 was reported by Horz et al. (2006) to range from 0.3 to $3.0 \mathrm{~g}$ per cc, which is too high for BCCA or BPCA dust particles. Also in comet $67 \mathrm{P}$, the morphology of the micron sized dust particles was recently reported by 
Bentley et al. (2016) to be very dense and that is again not consistent with BCCA or BPCA.

Owing to this we adopt an altogether different approach to generate highly fluffy / porous dust particles, where the bulk density can be 2.1 (for a typical $40 \%$ porous pyroxene dust as detailed in Section 5). However, the widely polydisperse nature of cometary dust particles ( as reported by Bentley at al. 2016, for comet 67P), can not be accommodated in our present paper, due to the practical limit on computational time. Also, the larger dust particles are fewer in numbers, hence have less influence on light scattering properties, so our calculations were not extended to particle sizes greater than one micron.

Again, Brownlee (1985) predicted on a very low density with regard to large ( 300 micron) dust particles, but such large dust particles are mostly not considered for modeling due to the reasons just explained above. Also for such large dust particles scientists use geometrical optics approximations (Muinonen et al. 1996).

Subsequently, after the work by Brownlee (1985), the existence of fluffy / porous dust particles in comets, interstellar medium and other astronomical environments were also predicted by astronomers from the analysis of ground-based data (Greenberg \& Hage 1990; Xing \& Hanner 1997; Kimura 2001; Kimura et al. 2006 etc.). Both laboratory and computer simulations of solar radiation scattered from comets have been carried out to understand the structures of cometary dust particles (Wurm \& Blum 1998; Gustafson \& Kolokolova 1999; Hadamcik et al. 2010, 2013, 2014; Xing \& Hanner 1997; Kimura 2001; Petrova et al. 2004; Tishkovets et al. 2004; Gupta et al. 2006; Kimura et al. 2006; Bertini et al. 2007; Kolokolova et al. 2007; Moreno et al. 2007; Das \& Sen 2011; Das et al. 2011 and others).

\section{On the definition of the porosity}

As a starting point of the problem, we consider the list, by order of importance, of the parameters relevant for characterizing the dust particle scattering profile.

The most important parameter is the total volume of the dust particle, that is the amount of matter interacting with the electromagnetic wave. This parameter was already highlighted in the Rayleigh theory, and has been written in various forms, worth mentioning under the form of the effective radius, $a_{e f f}$, defined by the equality between the total volume of the matter inside the dust particle, $V_{m}$, and the volume of the perfect sphere of radius $a_{e f f}$, that is:

$$
V_{m}=\frac{4}{3} \pi a_{e f f}^{3}
$$

Porosity is another parameter ubiquitous in the physics of the disordered small dust particles, but it is not yet clear whether the parameter is relevant or not for the light scattering process. The present study tries to put ideas in order about that quantity. The first question is the proper porosity definition. Actually, one should consider two cases:

- the closed porosity is the case where the dust particle can be defined as a compact volume with voids inside it. In this context, voids are called: 'pores'. The 
porosity, $f$, of a dust particle of volume $V_{m}$ is naturally defined as:

$$
f=\frac{V_{p}}{V_{m}+V_{p}}
$$

with $V_{p}$ the total volume of the pores. Note that $f$ depends only on the nondimensional ratio $V_{p} / V_{m}$ between the total volume of the pores and the volume of the matter inside a given geometric volume.

Condensation of droplets in which gas bubbles are dispersed gives an example of such porous dust particles. This is the case for ash produced by coal pyrolysis (Jiang 2006).

- the open porosity is the case where the outer surface irregularities are of same order as the overall size of the dust particle. Such open structures going from surface to inner places are sometimes called 'fjords' by reference to the long, narrow, inlets going into the coasts of Norway (Barabási \& Vicsek 1990). A generic example is the case of fractal particles. If $d_{f}<3$ denotes the fractal dimension of the matter distribution inside the particle, then the total volume of the matter inside a sphere of radius $R$ smaller than the overall radius of the dust particle, scales as $\simeq c\left(R / a_{o}\right)^{d_{f}}$, with $c$ a numerical constant and $a_{o}$ the typical size of the grains, the dust particle is made of. Then, generalizing the concept of pores to the general voids inside a meaningful geometrical sphere, the porosity, as defined by Eqn. (2), writes:

$$
f=1-c\left(\frac{R}{a_{o}}\right)^{d_{f}-3}
$$

Taking $R$ as the typical radius of the dust particle (example radius of circumscribing sphere, or radius of gyration), one notes that the porosity Eqn. (3) is size-dependent, and goes to 1 for the large dust particles since $d_{f}-3<0$. This is in opposition to the fractal dimension, $d_{f}$, which is characteristic of the process and not of the size of the dust particles.

Despite the embarrassing possible size-dependence of the porosity, one can argue that $f$ is a relevant parameter for light scattering. Indeed, $V_{m}$ is the amount of homogeneous matter surrounded by empty space, interacting with the light, and $V_{p}$ is similarly the amount of voids surrounded by homogeneous matter, interacting with the light (from Babinet's principle). Then, using $V_{m}$ and $f$ appears to be the most important shape parameters to categorize light scattering of finite dust particles.

The next step (not considered in the present work) would be to consider the various correlations either in the matter or in the void distribution.

\section{Models and Methods}

In the following, we will discuss the case of the closed porosity, leaving for a companion article the case of the open porosity. 


\subsection{Generation of random particles with closed porosity}

As our knowledge about cometary dust particle becomes more complete thanks to extensive observations and in-situ cometary missions, the very high-porosity of the dust particles ejected by comet nucleus, appears to be essential result. A simple way to model very porous dust particles is to use the Ballistic Cluster-Cluster Aggregation model (Wurm \& Blum 1998). This approach may be relevant as the BCCA model corresponds to general physical conditions possibly met in the cometary environment (Meakin 1983,1984). However, we have to be aware of a possible flaw: forcing the fractal particles to realize a given value of the particle porosity requires to fix the average radius of the particles, because of Eqn. (3). As already mentioned, the BCCA or BPCA model does not appear to be suitable to represent the typical cometary dust particles.

So here, we take the opposite point of view: we do not mind the fractal structure of the particles, but we fix the porosity of the particles to a given value. Taking a model of closed porosity, the value of $f$ is a constant whatever the size of the dust particle is. In other words, $V_{m}$ and $V_{p}$ are the two independent parameters of the model.

This class of model corresponds to the following process: outer layer of the comet nucleus becomes more and more porous through mechanisms which may include release of trapped gases (including gas sublimation), irradiation by solar radiation, interaction with solar wind, etc. Therefore, microscopic voids form naturally down to the penetration depth of heat and solar radiations. The dust particles are formed in a second process from the breakup of the porous layer by the gas escaping through the inner parts of the nucleus.

\subsection{Generation of the porous dust particles}

Considering the DDA method to obtain light scattering properties, one starts from a large number of electric dipoles regularly placed on the nodes of a cubic lattice. Two dipoles are said to be connected, if they are nearest neighbours. The first ensemble of $N$ dipoles is arranged initially in the shape of a sphere of radius $a$. Voids are generated according to the following procedure: one dipole is chosen randomly and removed. If this change leaves the ensemble of the remaining dipoles all connected (that is every couple of dipoles can be linked by a sequence of connected dipoles), one keeps the change. If it is not the case (removing that dipole leads to isolated dipole or isolated group of dipoles), then removal is canceled, the dipole is put back in its original position. Then, one removes another dipole. The process stops when the desired porosity is obtained. Previously, similar studies were carried out with the dipoles unconnected and hence, such aggregates were unrealistic (Vilaplana et al. 2006, 2011).

The test for connection of the ensemble of dipoles is realized through epidemic algorithm (Demers et al. 1987). More precisely: a dipole is chosen randomly. Then, the list of all the dipoles connected to it is calculated (infection). One considers all the dipoles in the list and do the same calculation, that is determining all the dipoles, not considered before, and connected to them (spreading). At the end of the process, one knows the list of all the dipoles connected to the very first dipole. If all the dipoles of the actual ensemble are in the final list, the ensemble is connected. Otherwise the 
ensemble is disconnected.

This model allows to generate spherical dust particles of definite porosity, taking into account cohesion of the structure. These particles are homogeneous in average because of the random removal process. At the end, most of the pores are totaly included inside the structure. Other pores impinge on the dust particle surface, but they are a few in number and not relevant (unlike the 'fjords' of a fractal structure). According to the definition by Eqn. 22, the final ensemble of dipoles contains $(1-f) N$ dipoles. A slice of a dust particle of porosity $f=50 \%$ is shown in the Fig.1.

\subsection{Use of DDA code and generation of light scattering different matrix elements for randomly porous aggregate}

In order to calculate the polarization and intensities of scattered radiation, we use DDSCAT 7.3 light scattering code. We find that this code has the desired level of flexibility in order to be applied on the porous aggregate structures that we have reported in the section above. Even if our approach is quite general and can be used similarly in many different situations, we will explore the possibility to apply this technique to cometray dust particles, since large and reliable data base is currently available.

\subsubsection{Range of the particle diameters}

The dust particles ejected from comets have a wide range of sizes. We take here the lower limit for the dust particles diameter: $0.01 \mu \mathrm{m}$ - that is the lower limit used for Halley and other types of comets in the past (Sen et al. 1991). Ideally the upper limit for size distribution can go up to several micrometers, according to conclusions from ground-based observations of recently studied comets, such as 67P and others (Hadamcik et al. 2010, 2013, 2014). Also, for the comet 67P, the on-board GIADA instrument during ROSETTA mission had detected the presence of large dust particles (Rotundi et al. 2015), as well as many porous dust particles.

For computational reasons, we limit here the upper bound of dust particle size distribution to $1.0 \mu \mathrm{m}$, in accordance with many other authors (Das et al. 2011 and references therein). Indeed, the size distribution is often represented as an inverse power law, $\nu(a) \sim 1 / a^{\alpha}$ with large index $\alpha$ values ranging from 1.8 to 3.8 (Das et al. 2011 ; Kolokolova et al. 1997, 2001 ; Mukai et al. 1987 ; Sen et al. 1991). Then, the very large particles are so infrequent that they are expected not to influence the averaged light scattering properties much.

However, one has to be aware that accurate fit of polarization profile may require to consider particles significantly larger than $1 \mu \mathrm{m}$, especially for the small ( side) scattering angles if the particles are weakly absorbing (Zubko 2013). According to Zubko (2013), the polarimetric response near back scattering is well reproduced by particles with radii up to $1.5 \mu \mathrm{m}$; whereas, the polarimetric response at side scattering require involving even larger particles, may be with radii up to $2.5 \mu \mathrm{m}$. 


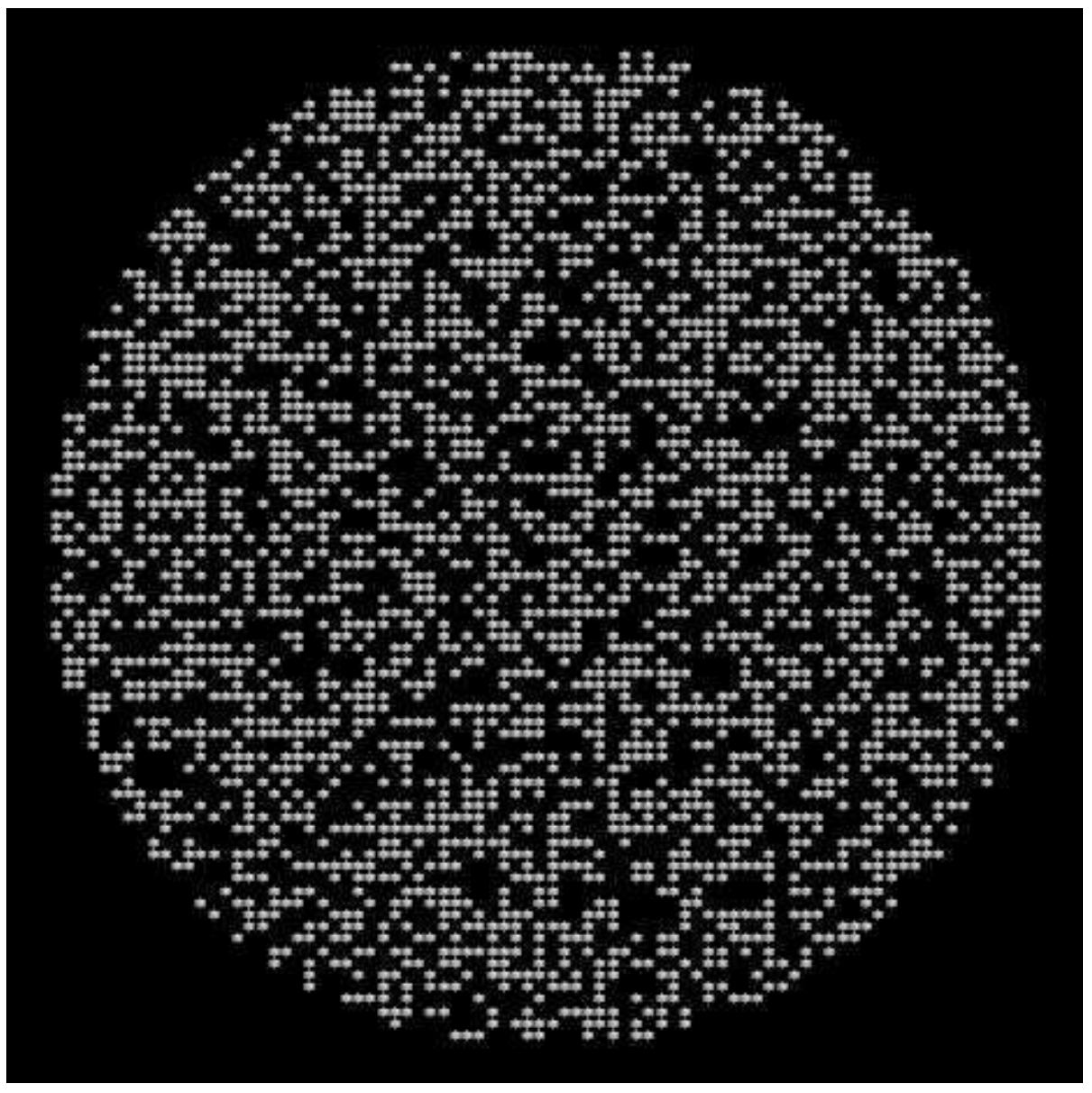

Figure 1: Slice of an aggregate structure generated by our code for the $50 \%$ porosity value. The width of the slice is 1 grain (dipole)-diameter. The slice passes through the geometric center of the particle. 
In our analysis below, we shall use the following values of the size-distribution exponent, $\alpha$, namely: $\alpha=1.8,2.8$ and 3.8, in order to check how the simulated values can match with the observed polarization data for comets.

\subsubsection{Composition of the particles}

For the composition of cometary dusts, we choose pyroxene with complex refractive index values as $(1.692,0.0492)$ at the wavelength $0.485 \mu \mathrm{m}$, and $(1.672,0.0185)$ at the wavelength $0.684 \mu \mathrm{m}$ (Das et al. 2011), with the justification for using this material given in Section 4. A large data base on cometary polarization for P/Halley is available at these two wavelengths.

Later, we extend our analysis to a more realistic two component composite ( pyroxene + organic) dust particle model.

\subsubsection{Computed optical parameters}

Running the DDSCAT 7.3 code allows to generate the Mueller matrix elements $S_{11}, S_{12}, S_{13}$ etc. needed to compute all the optical quantities, including light polarization (Bohren and Huffman 1983 ; Draine and Flatau 1994). The calculations are done performing averages over $10 \times 10 \times 10$ random orientations, and by integration over the dust particle size distribution. The linear polarization, $P$, is calculated using the relation:

$$
P=-\frac{S_{12}}{S_{11}}
$$

In principle, one should take into account the $S_{13}$ coefficient, using instead (Bohren and Huffman 1983):

$$
P=\frac{\sqrt{S_{12}^{2}+S_{13}^{2}}}{S_{11}}
$$

However, the random aggregates generated by our code have spherical symmetry in average, then we expect to have: $\left\langle S_{13}\right\rangle=0$ when averaged over angular randomization and the definition by Eqn. (4) can be used as a good approximation.

More precisely, difference between Eqns. (5) and (4) is a factor $\sqrt{1+\left(S_{13} / S_{12}\right)^{2}}$. In our present simulations, the highest $S_{13} / S_{12}$ value, that we encountered is $8.99 \%$. This has occurred for the $50 \%$ porosity dust particle at $\lambda=0.485 \mu \mathrm{m}$ and with power law index $\alpha=3.8$. This $S_{13} / S_{12}$ value should influence the estimated polarization value by a multiplicative factor of $\sqrt{1+(0.0899)^{2}}=1.004$. We are very much aware from observational astronomers that the error or uncertainty in observed polarization is never below $0.5 \%$, and thus a factor of $0.4 \%$ will have no effect on any simulation and modeling work. We also checked on some examples that both Eqns. (4) and (5) gave very similar values of the polarization. However, under a different situation, the linear polarization should be expressed by Eqn.(5).

\section{Milestones in the optics of porous particles}

It is now a long time that the problem of influence of the morphology of irregular dust particles on their optical properties has been addressed (e.g. Mishchenko, Hovenier and 
Travis 2000 and references therein). However, focus on porous dust particle - that is homogeneous dust particle with vacuum pores inside - in theoretical optics, is a much more recent approach.

\subsection{Irregular fractal aggregates}

In most of the astrophysical conditions, gases are so diluted that grain aggregation is expected to follow the Ballistic Aggregation procedure (Meakin 1983, 1984), that is all the clusters and grains move along random straight trajectories. According to the physical conditions, the process can be either successive sticking events of individual grains on aggregates (this is the Ballistic Particle-Cluster Aggregation model, BPCA), or diffusion is effective for all the clusters and the aggregates grow essentially sticking together (this is the Ballistic Cluster-Cluster Aggregation model, BCCA). The BPCA aggregates are compact in the fractal sense, that is their fractal dimension is equal to 3 , though they are porous, with very small pores. The BCCA aggregates are fractal with the fractal dimension $d_{f} \simeq 1.9$, then with big pores of the same order as the radius of the aggregate. Laboratory diagnostics of dust particle coagulation in the solar nebula suggests that the dust particles grow preferentially under the BCCA process (Wurm \& Blum 1998).

A variant of the BCCA is the Reaction-Limited Cluster-Cluster Aggregation model (RCCA). In the RCCA, sticking is hard to occur, for example because the aggregates are electrically charged. Then, two fractal clusters may randomly collide and interpenetrate because of their fluffy structures, while effective sticking is hindered by the electrostatic repulsions. The aggregates may stay long, entanged together. After some time, the thermal energy of the aggregates allow them to overcome the repulsive forces. It results in adhesion by van der Waals forces. The process leads to aggregates of fractal dimension $d_{f} \simeq 2.1$. In astrophysical clouds, BCCA or RCCA is expected to hold depending on whether grain electric charges are relevant or not.

But as discussed in Section 3.1, none of these aggregate structures can provide suitable models for cometary dust particles.

\subsection{Irregular compact aggregates}

As we are here interested in non-fractal shapes, we may recall that Shen et al. 2008, 2009 considered interesting modifications to standard BPCA in which the grains migrate along the surface of the aggregates by rolling or sliding over the first-contact grain (Ballistic Aggregation with Migration, BAM). These reorganizations produce even less porous aggregates than BPCA. Allowing for one (BAM1) or two (BAM2) migrations produce aggregates of decreasing porosity. Das et al. 2011 took help of this model along with BCCA to simulate observed cometary polarization data. The BAM models are not based on realistic rearrangement of the grains inside the dust particles, and they are used as a "cheap approach" (Seizinger \& Kley 2013) to generate aggregates denser than those obtained using the realistic BPCA process.

Other aggregation models were proposed for specific purposes. A famous example is the off-lattice Eden model, used to simulate tumor growth. In this model, grains are added randomly on any location tangent to a grain of the aggregate and realizing no 
overlaps with other grains. It results in compact structure with irregular surface and small pores inside.

Unlike the fractal dust particles, the compact-shape dust particles are not characterized by their fractal dimension (since it realizes the maximum value $d_{f}=3$ ), but by their porosity, that is the fraction of voids inside the particle. For instance, large BPCA aggregates porosity (fraction of voids inside the dust particle) is about $85 \%$, whereas the BAM2 aggregates are denser with porosity about $70 \%$. The porosity of Eden-model particles is about $35 \%$ (Wang et al. 1995).

\subsection{Porous particles}

Lumme \& Rahola (1994) had generated porous aggregate structures considering compact pseudo-sphere made up of many smaller spheres (dipoles), then removing some of the dipoles stochastically. They just did not remove too many dipoles, so that in the remaining structures all the dipoles remain connected.

They also considered a second approach where sub-volumes made of many dipoles were removed stochastically from the pseudo-spherical volumes, but without caring about the connectivity. Using DDA theory, the results from these porous aggregates were compared with same calculated by Mie and Effective Medium theories. The authors made detailed study to note agreement and disagreement under different situations.

Further, Wolff et al. $(1994,1998)$ studied the effect of porosity on the extinction and polarization for two types of porous dust particles: one with Rayleigh vacuum inclusions and the other one with non-Rayleigh vacuum inclusions. The authors considered compact cluster of dipoles within the original dust particle and the number of dipoles that was removed per cluster ( to create vacuum inclusions) controlled the amount of the porosity. The sizes of the clusters and their distribution played a major role in deciding the scattering properties.

Wolff et al. (1994) in their work on Rayleigh vacuum inclusions considered porosities up to $80 \%$ (with size parameters up to 10 ) and found that the $Q_{e x t}$ values are in good agreement between the DDA and Effective Medium Theory, barring a few exceptions. Considering aligned spheroidal dust particle they arrived at the same conclusions about the nature of polarization, with higher number of exceptions. Further works on the non-Rayleigh inclusions were published by Wolff et al. (1998) who considered porous spheres (with orientation randomized) and calculated the angular distribution of scattered intensities and polarization. The authors noted significant disagreement between the results from DDA and Effective Medium Theory calculations for non-Rayleigh inclusions, which however went down with polydisperse samples and obviously increased with size parameter and porosity.

Recently, Vilaplana et al. $(2006,2011)$ had addressed the problem of the effect of porosity on the polarization, $P$, and light scattering efficiency, $Q_{s c a}$. The authors generated dust particles with fluffiness/porosity by randomly removing dipoles, without constraining the remaining dipoles to stay connected. They defined the fluffiness degree as the percentage of dipoles removed, and generated aggregates with fluffiness degrees $15 \%, 25 \%$ and $50 \%$. As can be seen in the Figs. 9, 10 and 11 of Vilaplana et al. 
(2011), a displacement to the longer wavelengths and rising of the first maximum of $Q_{s c a}$ are observed as the fluffiness degree increases. The result is that both the real and imaginary parts of the effective refractive index decrease simultaneously as the fluffiness degree is increased. This behavior can be expected as the authors calculated the effective refractive index of the porous dust particle by the Maxwell-Garnett (1904) theory.

Other recent works made in this direction are worth mentioning here. The authors, Zubko et al. (2005), used a model of agglomerates based on random spatial distribution of blocks, each block being made either of array of dipoles or of void. Connectedness of the overall object is not considered. It results in specific disordered structures which might be relevant in the context of agglomerates of debris, such as in an ensemble of big solid particles experiencing many fragmentation events through collisions. Optical properties of these agglomerate debris were discussed using DDA method.

The main results were that the importance of the negative polarization branch at small phase angles decreases as the porosity increases (Zubko 2005). In particular, in the extreme cases of the fractal dust particles (RCCA or BCCA) for which the pores are essentially big - hence the matter distributed under the form of Rayleigh grains inside the particle -, the negative polarization branch is much more shallow than in the case of real cometary dust particle polarization. Moreover, there are only small differences between the debris in contact and the separated debris, provided the debris form an optically coherent system (Zubko 2008). In another work Zubko et al. (2015) conclude that, the optical features are fairly indifferent to the definite morphology of these agglomerates. The optical features being essentially the result of multiple scattering by the coherent solid fragments.

\section{Results and Discussions}

In the present paper, our approach is different from those discussed above, as we remove dipoles randomly, with the strict rule that no isolated dipole or cluster of dipoles is left. Thus, the removal process generates randomly inclusions of various sizes, which can be either Rayleigh or non-Rayeigh scatterers . The number of non-Rayleigh inclusions increase, in average, with the porosity. However, in another future work we want to concentrate specifically on non-Rayleigh inclusions in our model, as it will involve generation of new aggregate structure and a lot of computational work.

\subsection{The model of porous particle}

We consider connected aggregate structures with various porosities. We start with compact aggregate sphere where we have approximately 120,000 dipoles and then we generate structures with various porosity values $10 \%, 20 \%, 30 \%, 40 \%$ and $50 \%$. While running DDSCAT code, we do averaging over $10 \times 10 \times 10$ random orientations. We use the power law size distribution with lower bound and upper bound as 0.01 and $1.0 \mu \mathrm{m}$ as discussed earlier. We perform integration over this size range numerically, by doing the calculation in 71 steps and then using Simpson's 1/3 rule. The index for power law dust size distribution is considered as 1.8, 2.8 and 3.8 as these are the 
most commonly used values in literature (Das et al. 2011 and references therein). As far as, the compositions are concerned, there are naturally many possible options, and scientists often consider mixtures of various types. But since we want here to focus our studies on porosity, we first consider only one type of composition which is pyroxene marked by the complex refractive index values of $(1.692,0.0492)$ and $(1.672,0.0185)$ at the wavelengths $\lambda=0.485 \mu \mathrm{m}$ and $0.684 \mu \mathrm{m}$ respectively (Das et al. 2011). This material is most commonly used as a candidate for cometary dust.

In a second step, we consider a more realistic composite model for cometary dust particles as described by Das et al. 2011, where the authors fitted, the mixing ratio between silicates (pyroxene) and organics to be 78:22 by volume. Das et al. (2011) adopted the refractive indices of organics from Jenniskens (1993) which are (1.842, $0.459)$ at $0.485 \mu \mathrm{m}$ and $(1.942,0.357)$ at $0.684 \mu \mathrm{m}$. Such a pyroxene particle with 40 $\%$ porosity will have bulk density $2.1 \mathrm{~g}$ per cc, as density of pyroxene is mostly reported to be 3.5 in the literature. However, with $22 \%$ organic inclusions this value will go down slightly. So these density values will be similar to what has been reported for comet Wild2 (Horz et al. 2006). However, we note here that there are several observational evidences, which suggest that cometary dust particles are heterogeneous and have inclusions of organics, iron sulfides and oxides etc. For example, dust emission features observed in comets is very wide and are suggestive mixtures of many compounds (Hanner \& Bradley, Comets II 2004). If we consider a composite model with all these constituents, the calculations done in the present work will become very lengthy, so we limit the calculations below to the model by Das et al. (2011).

\subsection{Effects of the porosity of aggregates on polarization profile}

In this section we discuss specifically how the porosity, $f$, of cometary dust particles affects cometary polarizations.

It was noticed by Das et al. 2011 (and references there in) that the linear-polarization curve of the Halley's comet is bell-shaped - which is a general feature among all the comets -, with max-value, $\left(P_{\max }\right)$, and min-value, $\left(P_{\min }\right)$, about $25 \%$ and $-5 \%$ respectively. However, these values are not very accurate, since it was not possible to make polarization measurements at all the scattering angles. We should also note that $P_{\max }$ can be even higher than $25 \%$ and $P_{\min }$ even more negative than $-5 \%$. Crossover from positive to negative polarization values takes place at a scattering angle $\simeq(155-160)$ degrees.

In Figures 2-7, we reproduce the calculated (simulated) polarization values for the two-components porous dust particle model, along with the values which were previously calculated for the single component pyroxene model. As expected, these two plots differ and exhibit different $P_{\max }, P_{\min }$, etc values. In Table 1 , we list certain porosity values with the corresponding $P_{\max }$ (in $\%$ ), $P_{\min }$ (in \%) values and the crossover angle (in degrees) between the positive and the negative polarization branches, for the bell-shaped polarization curves, calculated for pyroxene and (pyroxene+organic) dust particle. We also reproduce inside bracket $(\cdots)$, the scattering angle values at which these $P_{\max }$ and $P_{\min }$ occur. Only those simulated values are tabulated which are close to the observed polarization features of comet Halley.

More precisely, one can see on the Figures 2-7 that varying the porosity values 
(namely: from $f=0 \%$ to $f=50 \%$ ) both the maximum and minimum polarization values change, along with their positions. However, the behavior is complicated, as the negative polarization branch becomes deeper with the increase in porosity and then again it becomes shallower. This non-monotonic phenomenon appears for both the wavelengths $0.485 \mu \mathrm{m}$ and $0.684 \mu \mathrm{m}$, and with the three values of power law index $\alpha=1.8,2.8$ and 3.8 .

Among all the different sets of parameters considered here, the matching with the observed polarization data of comet is best obtained with $40 \%$ porosity and $\alpha=3.8$ power law index at both the wavelengths for pyroxene model. For the composite model, also at $(\alpha, f)$ values equal to $(2.8,40 \%),(3.8,40 \%)$ and $(3.8,50 \%)$, we get bell shaped curves which look similar to the curve observed for comets.

In Fig. 8, we provide the plots for the simulated polarization curves with $(\alpha, f)$ value fixed at $(2.8,40 \%)$ for pyroxene and for the (pyroxene+organics) composite model at $0.485 \mu \mathrm{m}$. For making this plot, the wavelength $0.485 \mu \mathrm{m}$ was chosen as highest number of observational data points exist corresponding to this wavelength. We clearly see that the observed data points lie between the two curves corresponding to pyroxene and (pyroxene+ organics). We may note here that Das et al. (2011) fixed this ratio at 78:22 between pyroxene and organics for the composite model, while using a combined (BCCA + BAM) model by curve fitting. So for the present aggregate model, this ratio can be again fine tuned. And it is obvious from the plot that, if the relative composition of organics is lowered from $22 \%$, then we will obtain closer fit to the data. However, this would require additional computational work, that we postpone for future.

In Table 1, we list only those simulated polarization values which are close to the above observed polarization values of $P_{\max }, P_{\min }$ and consistent with crossover angles. We should note here that, knowing exactly the observed values of $P_{\max }$, and $P_{\min }$ is difficult as the turning around the $P_{\max }$ and $P_{\min }$ values in the observed polarization curve has not ever been detected yet. One can only put lower limits on the $P_{\max }$ and $P_{\min }$ values.

At this stage one can now, fine tune the porosity and power law index values, and try to match the observed polarization data more closely. By a $\chi^{2}$ minimization technique, one can find the optimum values of porosity $(f)$ and power law index $(\alpha)$, which realize the best fit to the observed polarization data. As mentioned earlier, this we postpone for future, which will be a more detailed work.

Way back in 1987, immediately after the apparition of comet Halley, Mukai et al. (1987), carried out simulation work by varying the refractive index (that is composition) of cometary dust. As one can see from the Fig. 1 of their article, various shapes for the polarization versus scattering-angle curve are possible, as we change the refractive index of cometary dust. In the present work, we can see in a similar way that various shapes for the polarization versus scattering-angle curve are possible by changing the porosity $f$. These two results may be related since an effective refractive index can be defined dependent on the actual porosity using the Maxwell-Garnett effective medium theory. However, this procedure is empirical and it will be discussed in more details below in the Section 6. On the other hand, the procedure developed in the present work, 
provides a definite and analytical way of dealing with porosity of dust particle.

But what appears clear from the above analysis is that the present model does not result in appreciable differences in polarization values as compared to EMT.

\subsection{Effect of the porosity on the color}

In addition to the polarization features, comets also show interesting properties of photometric color. Since, there are many cometary molecules having their own emission bands within the visible region, we have to observe the comets only through some specific wavelength bands, where we can measure the light scattered by cometary dust alone and uncontaminated by cometary molecular emissions. Below we calculate 'color' values, so that we can compare it with observed 'color' values as available in literature ( example : Kolokolova et al. 1997).

The ratio of the scattered intensities as observed through such two continuum bands, when expressed in suitable log scale is called color. Kolokolova et al. (1997) had worked on cometary color and elaborated on the definition and the concept of cometary color. As stated by the authors, the color, $C$, can be suitably defined as :

$$
C=-2.5 \log \frac{I(0.485)}{I(0.684)} .
$$

The quantity $I(0.485)$ and $I(0.684)$ are the two recorded intensities of light scattered by cometary dust particles through the two wavebands centered around $0.485 \mu \mathrm{m}$ and $0.684 \mu \mathrm{m}$ respectively. In other words, cometary color can be measured simply by the relation:

$$
C=B(0.485)-R(0.684),
$$

where the $\mathrm{B}(0.485)$ and $\mathrm{R}(0.684)$ are the cometary magnitudes observed through the above cometary filters, which were standardized by International Halley Watch (IHW) and widely used for cometary photometry.

The color, $C$, can be estimated theoretically using Eqn. (6) by considering certain specific cometary dust population. The detailed procedure has been described by Kolokolova et al. (1997). From their Equations (9) and (10), we arrive at the following two equations below, which will be applicable directly in our case:

$$
\begin{aligned}
& I(0.485)=\int_{a \min }^{a \max } S_{11}(0.485, a) \frac{d a}{a^{\alpha}} . \\
& I(0.684)=\int_{a \min }^{a \max } S_{11}(0.684, a) \frac{d a}{a^{\alpha}} .
\end{aligned}
$$

where $S_{11}$ is the 'total intensity scattering matrix' element obtained after running DDSCAT code at the given wavelengths $0.485 \mu \mathrm{m}$ and $0.684 \mu \mathrm{m}$ and for the given dust particle radius $a$ expressed in $\mu \mathrm{m}$. The dust particle size follows the power law distribution $\nu(a) \sim 1 / a^{\alpha}$. The lower and upper limit of dust size distributions are $a_{\min }$ and $a_{\max }$ are respectively $0.01 \mu \mathrm{m}$ and $1.0 \mu \mathrm{m}$ in the present case. 
Here, the color values are calculated from the simulation data using the Eqns. (6), (8) and (9) for different porosity values of cometary dust particles, for the composition pyroxene and (pyroxene +organic). The colors are plotted against the scattering angles on the Figs. 9, 10 and 11 for different power law indices. Looking at these figures, we find that the color values, $C$, remain almost constant for different scattering angles at a given porosity, except that there is a sudden increase in the domain of the negative branch of the polarization curve (that is for scattering angles $>160$ degrees). Still more remarkable, the constant value of the color with the scattering angle does not change appreciably for the different porosities at a given power index value. One can conclude from these remarks that the color of the cometary dust depends essentially on the size-distribution of the dust particles and not on their porosity, with the noticeable exceptions for the backward scattering directions.

This is in confirmation of previous conclusions by Kolokolova et al. (1997). Indeed, Kolokolova et al. $(1997,2001)$ using power law index $\alpha=3$ or still higher, concluded that the cometary color is mainly determined by the size distribution of the cometary dust, whereas the cometary polarization is mainly determined by the complex refractive index. Similar conclusion was drawn in the recent results by Zubko et al. (2015) about polarization ratios of other kinds of agglomerates, with power-law exponent $\alpha$ between 1 and 4 .

However, we cannot explain quantitatively at the same time both the polarization curve of the comet Halley and its color. Indeed, the average color of the comet Halley is known to be $C \simeq-0.024$ (Kolokolova et al. 1997), which is a value much smaller than the color found in the present work for the appropriate values of $\alpha$ and $\mathrm{f}$.

The estimated color values with pyroxene are the $\simeq-0.2, \simeq-0.6$ and $\simeq-1.0$ respectively for the three power law index values $\alpha=1.8,2.8$ and 3.8. And the corresponding values for the composite model are very similar, except that at high porosities the values corresponding to composite model is slightly higher. Now the observed color values should be always corrected for the offset introduced by solar color itself. This offset has been estimated to be around 1.106 for a paricular solar analogue under certain observing conditions as reported by A'Hearn et al. (1984). So if the values reported by Kolokolova et al. (1997) is not already corrected by solar color 'offset', then we see our calculated color values for power law index 3.8, is very close to what has been reported by Kolokolova et al. (1997) from observations. Otherwise, the $\operatorname{color}(C)$ values theoretically estimated by us found to be much more negative than the observed Halley's comet color. A possible interpretation of the numerical data is that big dust particles (more numerous for the small values of $\alpha$ ) tend to be white, whereas small-size dust particles (dominant for the large values of $\alpha$ ) are essentially reddish. Therefore, the number of large particles may well have been underestimated compared to the real Halley comet dust particle size-distribution. Larger value of the size cutoff $a_{\max }$ should be tempted, but that means adding one more free parameter to the model, then making the simulations and their analysis much more lengthy. Let us note that, until now, there have been no successful attempts where a single dust model could explain both polarization and color.

In the above, we estimated color values, in order to compare with the observed 
values reported by Kolokolova. In literature more often 'color slope' is used rather than 'color', to understand the reddening introduced by dust paricle (A'Hearn et al. 1984). Towards this we have calculated the 'color slope', for the Pyroxene and (Pyroxene+ Oragincs) dust particle, for power law index $=2.8$ ( this value seems to be good choice from the matching of polarization curve) and reproduced in Figure 12, for comparison.

\section{Relevance of the Effective Medium Theory}

As mentioned in the previous Sections and also discussed by Maxwell-Garnett (1904), the effect of porosity, $f$, of dust particles can be approximately represented by replacing the actual matter refractive index $n=(m, k)$ by an equivalent homogeneous matter of refractive index $n^{\prime}=\left(m^{\prime}, k^{\prime}\right)$. Such phenomenological approach is known as Effective Medium Theory (EMT). This has been shown to be an accurate approximation for "discrete random media", that is: a large number of small inclusions distributed quasirandomly in a volume (Mishchenko et al. 2014). It was even more precisely proved to be a rigorous approach in the limit of uncorrelated small inclusions (Mishchenko et al. 2016a).

The present work investigates particles a step further in the complexity. Indeed, we are dealing with particles for which inner space-correlations are relevant and inclusions are not infinitely small. It makes the EMT to be an approximation of the real electromagnetic scattering process, valid in some ranges of the optical parameters, but exhibiting distinctive differences in other ranges. Various schemes are available for determining effective refractive index, among the most recent ones which will be worth mentioning here include the work by Mishchenko and his co-workers (Mishchenko et al. 2016a, 2016b).

Following Voshchinnikov et al. (2007), we adopt here the mixing rule of Bruggeman, since it has been found to work for wide porosity range and deviations from the exact results do not exceed $5 \%$.

This symmetric mixing rule is stated below with our set of symbols :

$$
(1-f) \frac{n^{2}-n^{\prime 2}}{n^{2}+2 n^{\prime 2}}+f \frac{1-n^{\prime 2}}{1+2 n^{\prime 2}}=0 .
$$

Solving this complex-value equation allows to compute the effective refractive index for any set of the parameters $\{f, m, k\}$. Then, using the Mie result for the homogeneous spheres with radii distributed as $\nu(a)$, approximate averaged polarization and color of the ensemble of porous particle characterized by $\{f, m, k\}$ and $\nu(a)$ can be calculated. This scheme was used previously for polarization (viz. Sen et al. 1991).

The values of the effective refractive index are shown in the Table 2 . The corresponding polarization and color values are plotted along with those obtained previously using porous aggregates and DDA code in the Figs. $2-7$ and 9-11. When comparing between EMT ( Mie) and pyroxene ( DDA) dust particles, it is clear that the Effective Medium Theory can not exactly reproduce the numerical polarization and color values obtained using DDA method on the simulated porous aggregates, though most of 
wavelength $=0.485 \mu \mathrm{m}$, power index $=1.8$
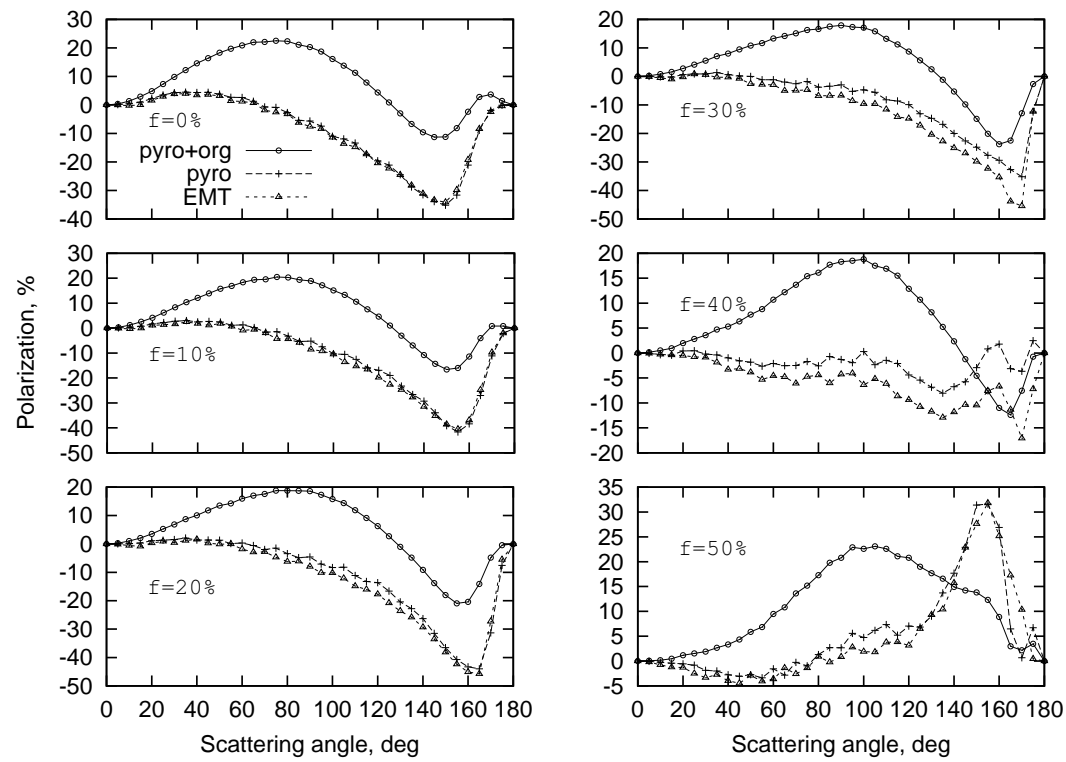

Figure 2: Variations of polarization with the scattering angles expressed in degrees for various values of dust particle porosities. The polarization has been calculated for particles having size range $0.01 \mu \mathrm{m}-1.0 \mu \mathrm{m}$, with a power-law size index 1.8 and wavelength $\lambda=0.485 \mu \mathrm{m}$. The curve marked by ' $\triangle$ ' is the one drawn with the EMT theory. The curve marked by ' + ' is the one drawn with the DDA for Pyroxene and the one marked by 'o' is the one drawn with the DDA for (Pyroxene + Organics)

the data are comparable. The differences become more noticeable, as the porosity increases. Vaidya et al. (2007) had also observed similar limitations of Effective Medium Theory.

In our present work, we studied the effect of dust particle porosity on polarization and colors (includes intensity). To do this we considered a dust particle composition model, which is more realistic and has been already worked out to simulate the polarization data for comet Halley by other authors ( Sen at al. 1991, Lasue et al. 2009, Das et al. 2011 etc.). But in the present work, we must note that, our aim is just to grossly match the simulated ( or theoretically calculated) values with the polarization and color values that were observed for a well known comet Halley. We also note that, we can roughly match the polarization data with a dust particle population having densities close to what was reported by in situ observations and this density matching was not earlier possible with BCCA model, which is often used for cometary modeling work. 
wavelength $=0.485 \mu \mathrm{m}$, power index $=2.8$
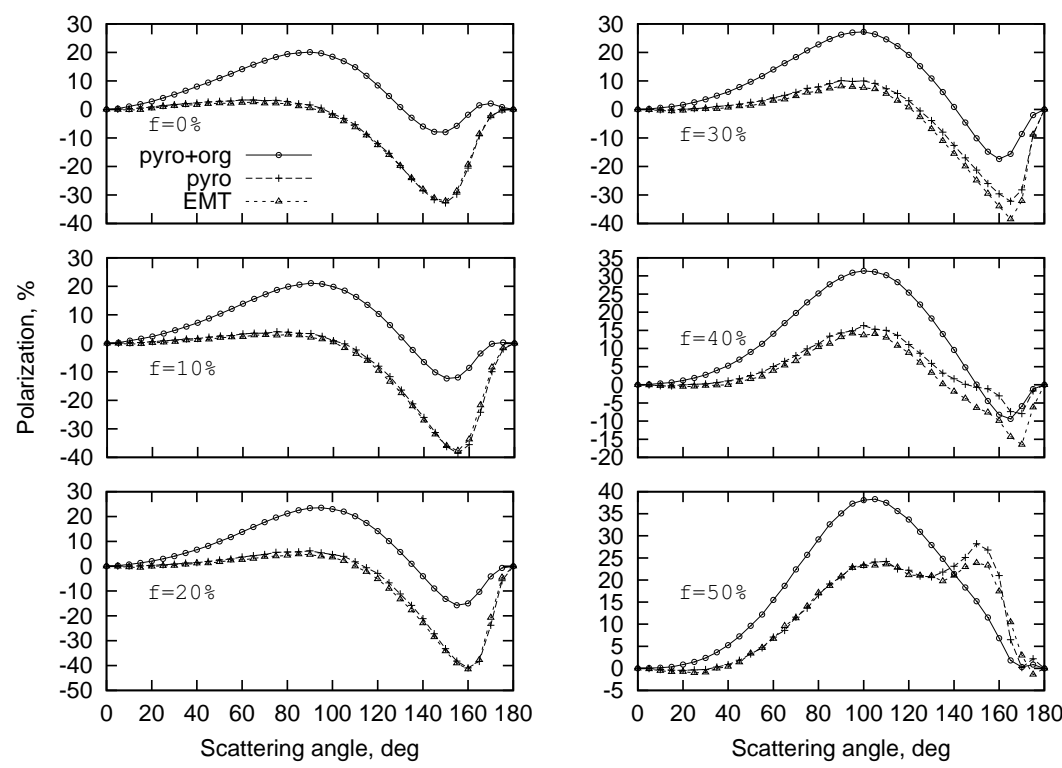

Figure 3: Variations of polarization with the scattering angles expressed in degrees, for various values of dust particle porosities. The polarization has been calculated for particles having size range $0.01 \mu \mathrm{m}-1.0 \mu \mathrm{m}$, with a power-law size index $\alpha=2.8$ and wavelength $\lambda=0.485 \mu \mathrm{m}$. The curve marked by ' $\triangle$ ' is the one drawn with the EMT theory.The curve marked by ' + ' is the one drawn with the DDA for Pyroxene and the one marked by 'o' is the one drawn with the DDA for (Pyroxene + Organics)

Table 1: List of certain porosity values with the corresponding simulated $P_{\max }$ (in \%), $P_{\min }$ (in \%) values and the crossover angle (in degrees) between the positive and the negative polarization branches, from bell-shaped polarization curves for comet. The scattering angles at which maximum and minimum of polarization occur are recorded within (...) bracket. These values are calculated for Pyroxene and composite (pyroxene+organic) dust particle. Only those simulated values are tabulated which are close to the observed polarization values.

\begin{tabular}{lccccr}
\hline material & $\lambda(\mu \mathrm{m})$ & porosity $f$ & $P_{\max }($ angle $)$ & $P_{\min }($ angle $)$ & crossover angle \\
\hline pyroxene & 0.485 & $40 \%(2.8)$ & $16(\sim 100)$ & $-8(\sim 170)$ & 160 \\
& 0.485 & $40 \%(3.8)$ & $45(\sim 100)$ & $-8(\sim 170)$ & 157 \\
& 0.684 & $40 \%(3.8)$ & $40(\sim 100)$ & $-8(\sim 170)$ & 158 \\
composite & 0.485 & $40 \%(2.8)$ & $32(\sim 100)$ & $-8(\sim 165)$ & 155 \\
\hline
\end{tabular}



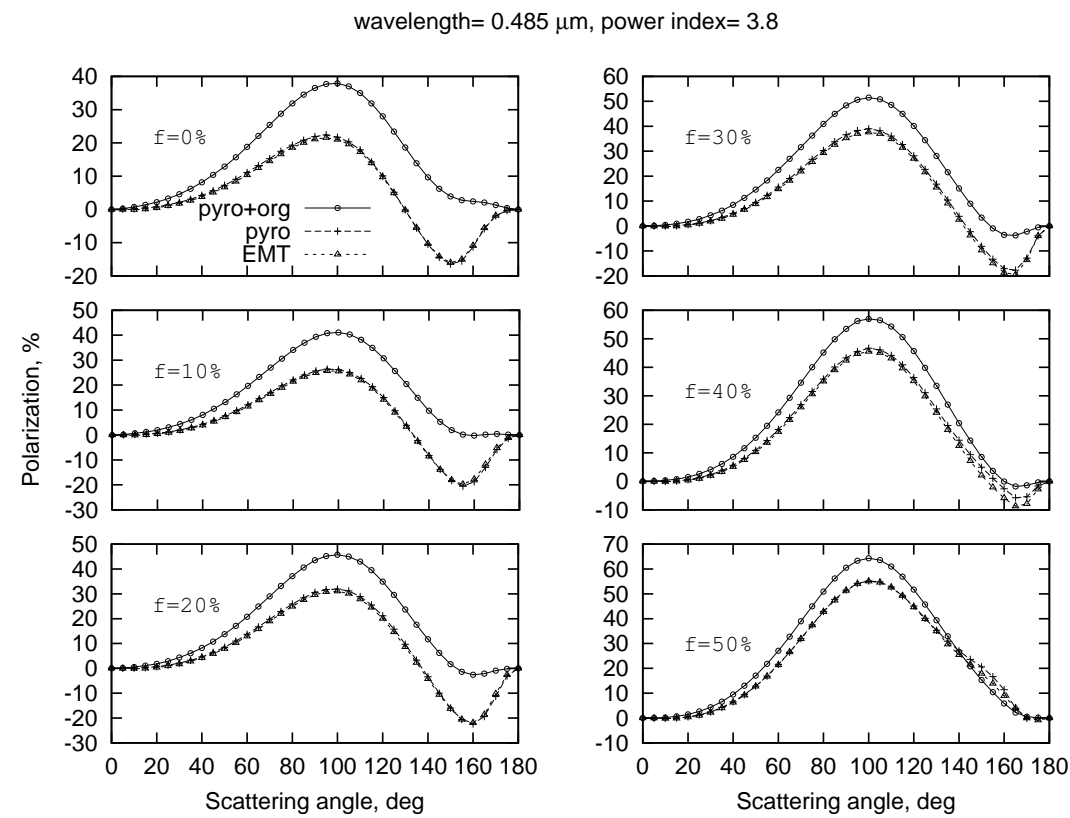

Figure 4: Variations of polarization with the scattering angles expressed in degrees, for various values of dust particle porosities. The polarization has been calculated for particles having size range $0.01 \mu \mathrm{m}-1.0 \mu \mathrm{m}$, with a power-law size index $\alpha=3.8$ and wavelength $\lambda=0.485 \mu \mathrm{m}$. The curve marked by ' $\triangle{ }^{\prime}$ ' is the one drawn with the EMT theory. The curve marked by ' + ' is the one drawn with the DDA for Pyroxene and the one marked by 'o' is the one drawn with the DDA for (Pyroxene + Organics) 

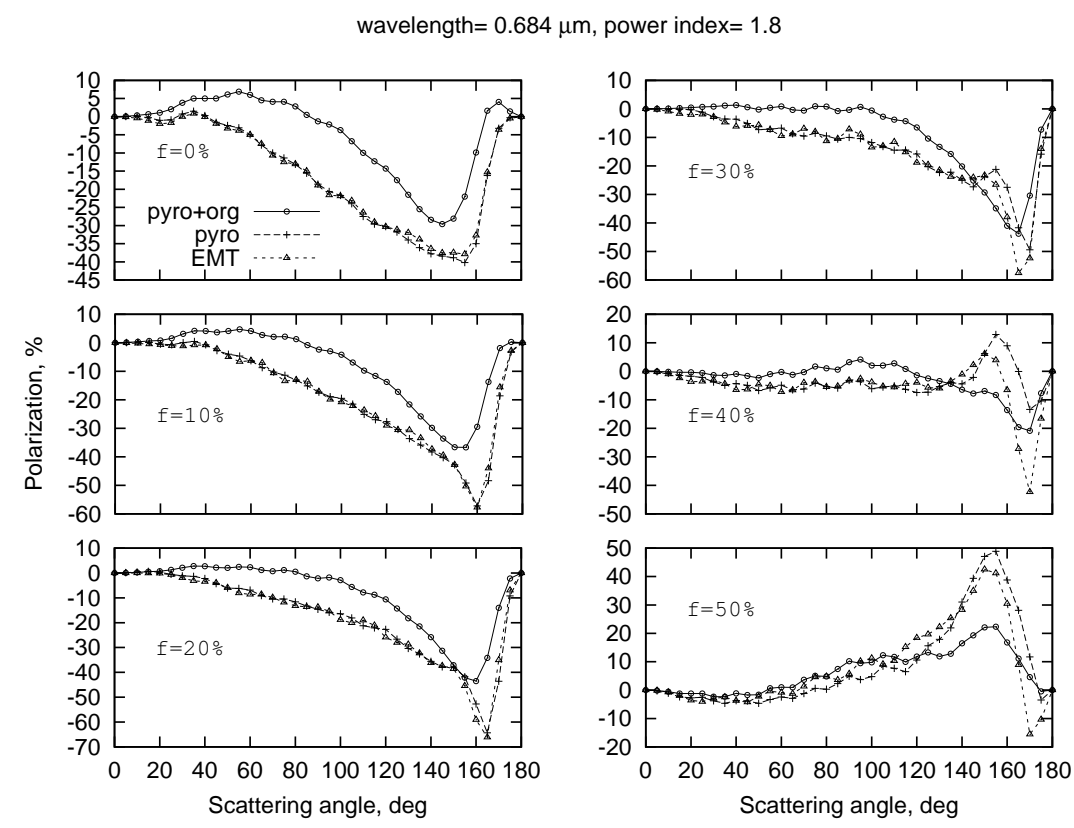

Figure 5: Variations of polarization with the scattering angles expressed in degrees, for various values of dust particle porosities. The polarization has been calculated for particles having size range $0.01 \mu \mathrm{m}-1.0 \mu \mathrm{m}$, with a power-law size index $\alpha=1.8$ and wavelength $\lambda=0.684 \mu \mathrm{m}$. The curve marked by ' $\triangle$ ' is the one drawn with the EMT theory. The curve marked by ' + ' is the one drawn with the DDA for Pyroxene and the one marked by 'o' is the one drawn with the DDA for (Pyroxene + Organics) 

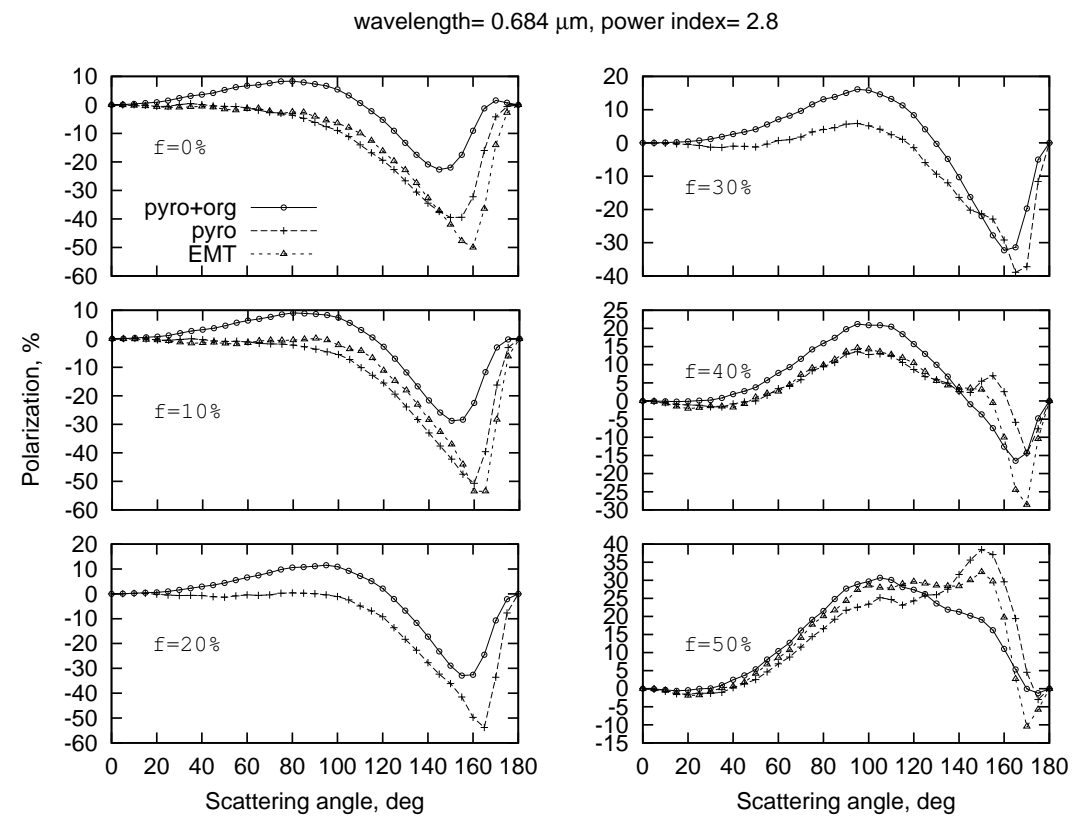

Figure 6: Variations of polarization with the scattering angles expressed in degrees, for various values of dust particle porosities. The polarization has been calculated for particles having size range $0.01 \mu \mathrm{m}-1.0 \mu \mathrm{m}$, with a power-law size index $\alpha=2.8$ and wavelength $\lambda=0.684 \mu \mathrm{m}$. The curve marked by ' $\triangle{ }^{\prime}$ ' is the one drawn with the EMT theory. The curve marked by ' + ' is the one drawn with the DDA for Pyroxene and the one marked by 'o' is the one drawn with the DDA for (Pyroxene + Organics) 

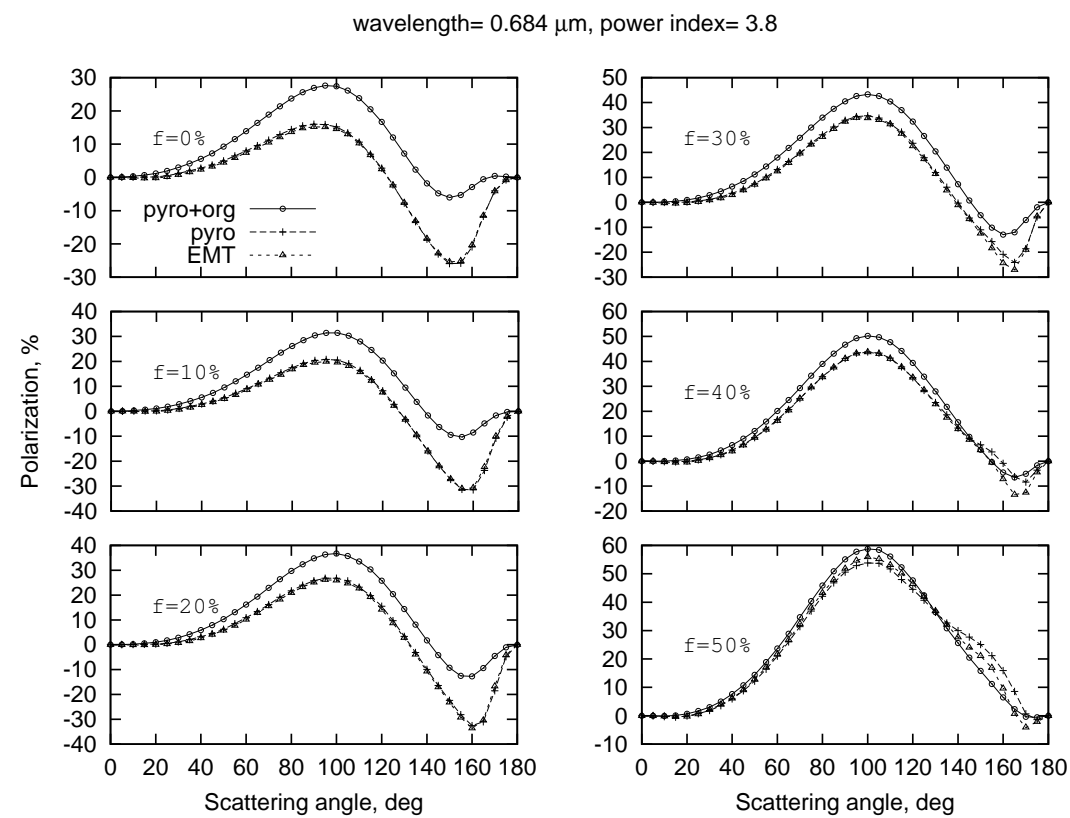

Figure 7: Variations of dust polarization with the scattering angles expressed in degrees, for various values of dust particle porosities. The polarization has been calculated for particles having size range $0.01 \mu \mathrm{m}-1.0 \mu \mathrm{m}$, with a power-law size index $\alpha=3.8$ and wavelength $\lambda=0.684 \mu \mathrm{m}$. The curve marked by ' $\triangle$ ' is the one drawn with the EMT theory. The curve marked by ' + ' is the one drawn with the DDA for Pyroxene and the one marked by 'o' is the one drawn with the DDA for (Pyroxene + Organics). 


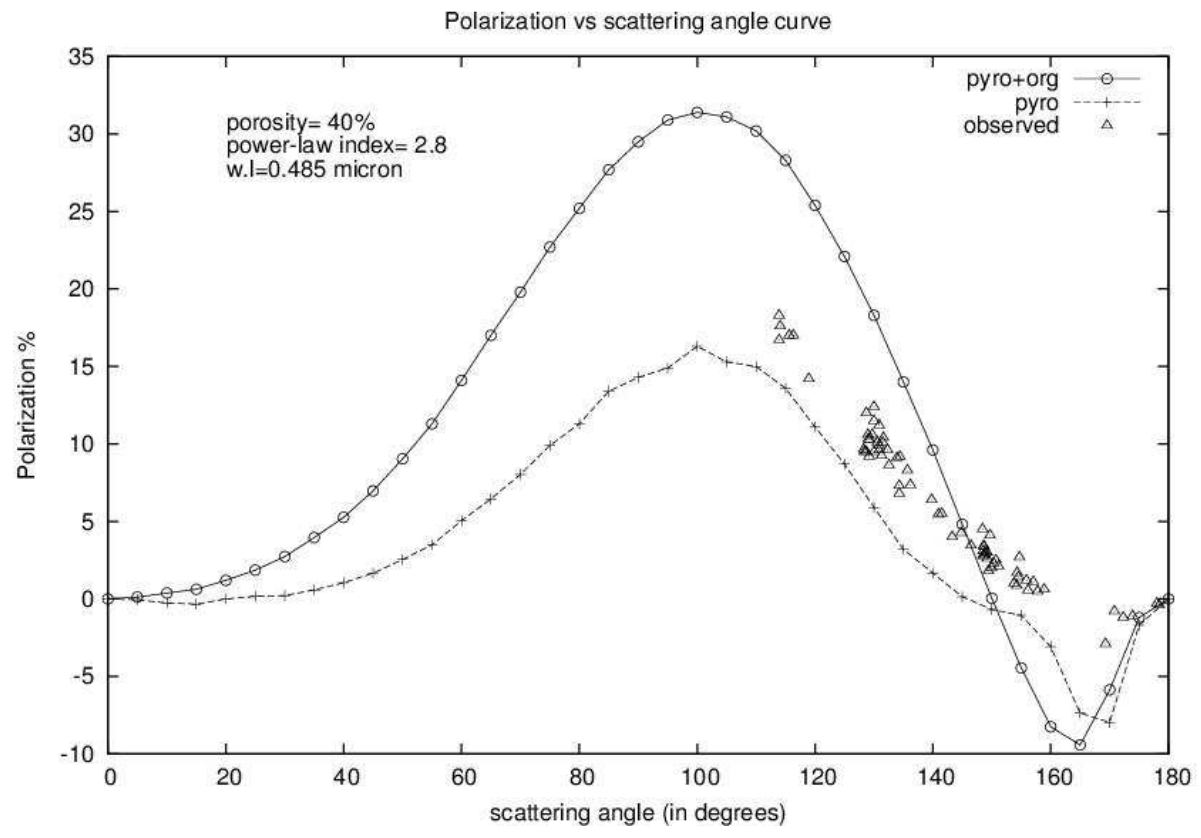

Figure 8: Variations of the simulated polarization versus the scattering angle for the two compositions pyroxene and (pyroxene + organic), with $(\alpha, \mathrm{f})$ values fixed at $(2.8$, $40 \%$ ), at wavelength $0.485 \mu \mathrm{m}$. The observed polarization values of comet Halley at same wavelength are shown along with. 

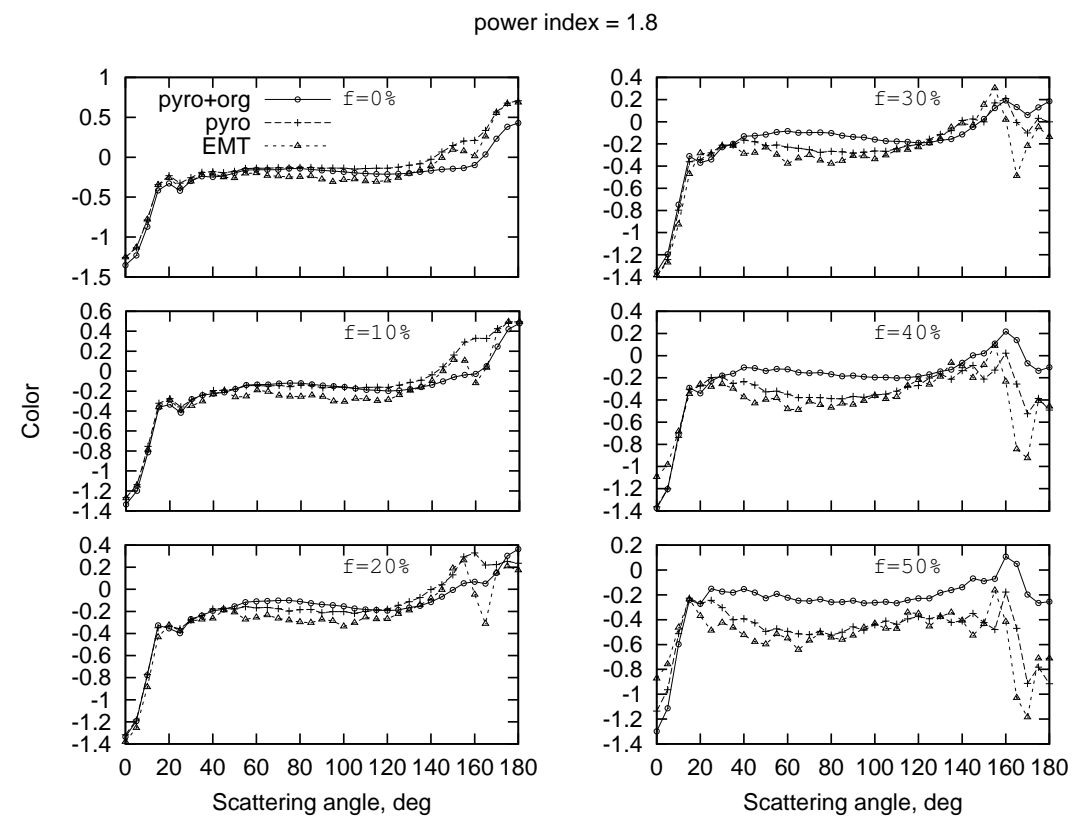

Figure 9: Variations of the color with the scattering angles expressed in degrees, for various values of dust particle porosities. The color has been calculated for particles having size range $0.01-1.0 \mu \mathrm{m}$, with a power-law size index $\alpha=1.8$. The curve marked by ' $\triangle$ ' is the one drawn with the EMT theory. The curve marked by ' + ' is the one drawn with the DDA for Pyroxene and the one marked by 'o' is the one drawn with the DDA for (Pyroxene + Organics) 

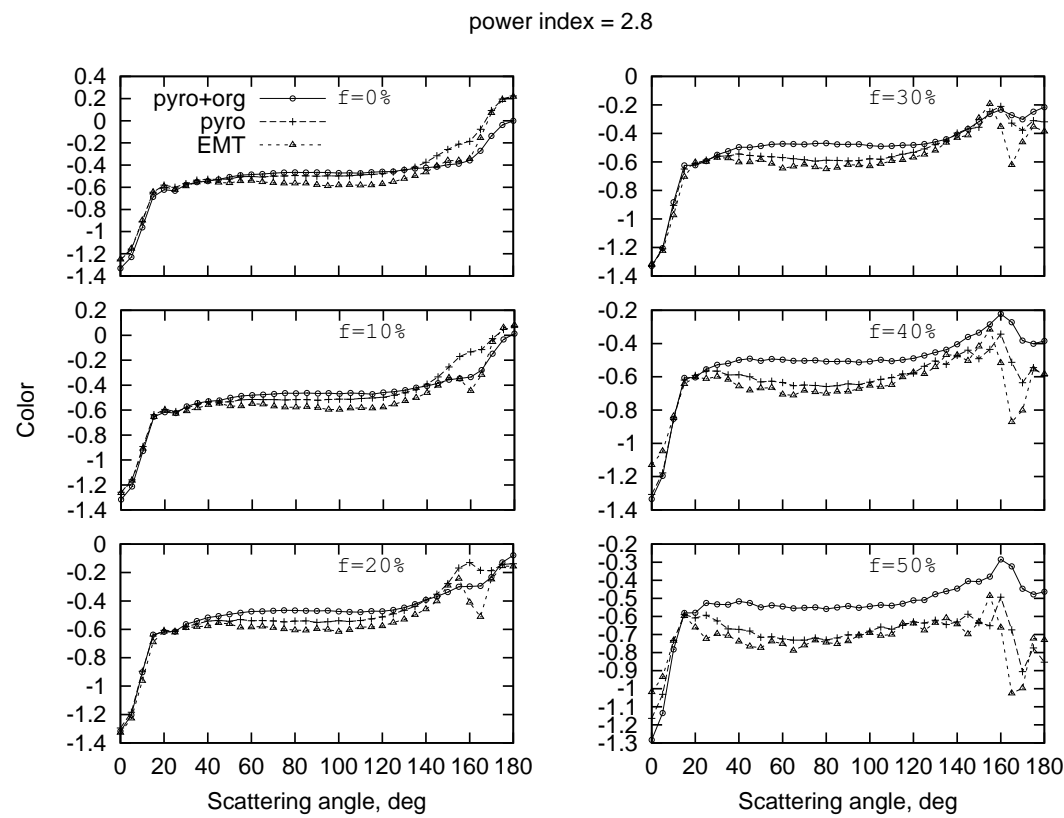

Figure 10: Variations of color with the scattering angles expressed in degrees, for various values of dust particle porosities. The color has been calculated for particles having size range $0.01-1.0 \mu \mathrm{m}$, with a power-law size index $\alpha=2.8$. The curve marked by ' $\triangle$ ' is the one drawn with the EMT theory. The curve marked by ' + ' is the one drawn with the DDA for Pyroxene and the one marked by 'o' is the one drawn with the DDA for (Pyroxene + Organics) 

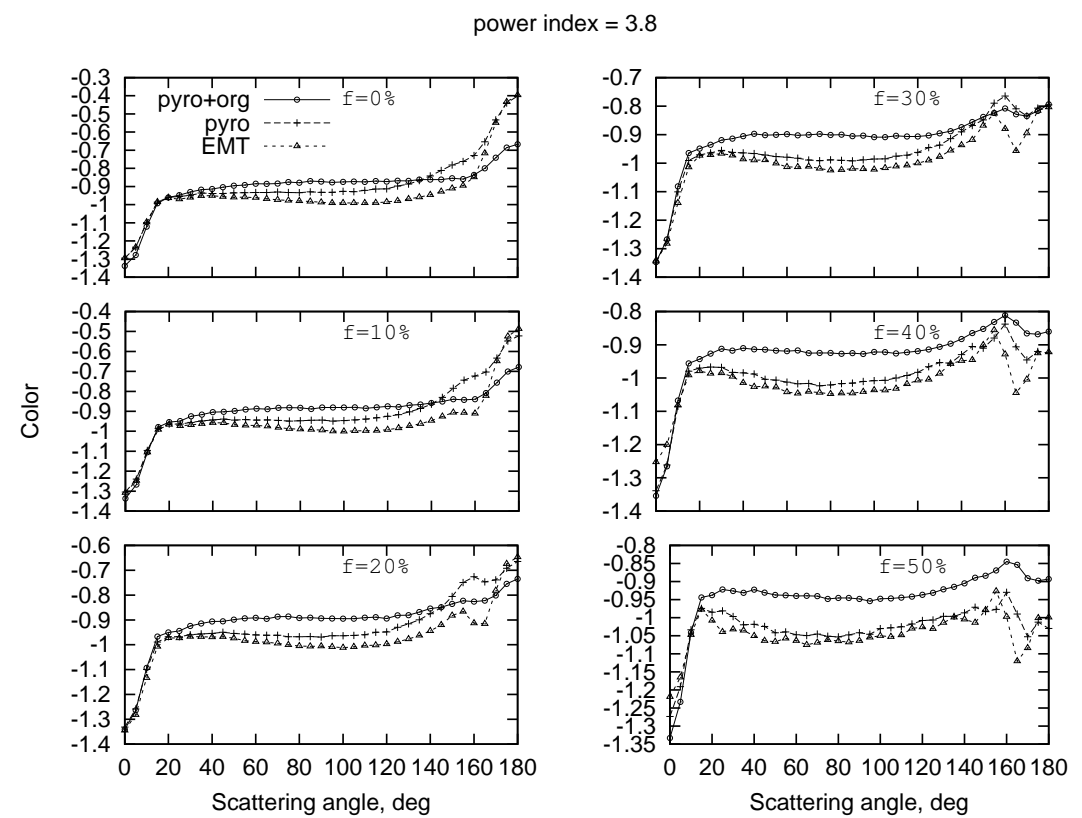

Figure 11: Variations of color with the scattering angles expressed in degrees, for various values of dust particle porosities. The color has been calculated for particles having size range $0.01-1.0 \mu \mathrm{m}$, with a power-law size index $\alpha=3.8$. The curve marked by ' $\triangle$ ' is the one drawn with the EMT theory. The curve marked by ' + ' is the one drawn with the DDA for Pyroxene and the one marked by 'o' is the one drawn with the DDA for (Pyroxene + Organics). 

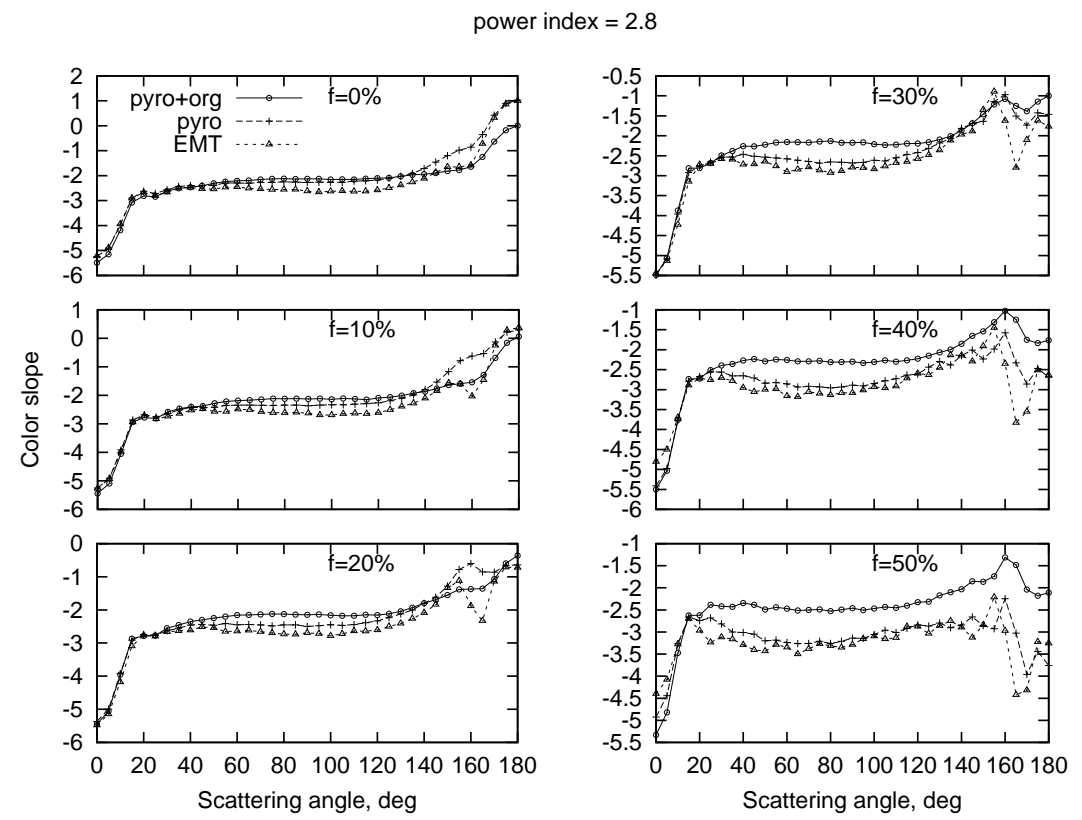

Figure 12: Variations of 'color slope' ( expressed in unit of per $\mu \mathrm{m}$ ) with the scattering angles, for various values of dust particle porosities, with $(\alpha, \mathrm{f})$ fixed at $(2.8,40 \%)$ calculated for pyroxene and (pyroxene+organic) model. 
Table 2: The complex Effective Refractive Index $\left(m^{\prime}, k^{\prime}\right)$ values as calculated by using Effective Medium Theory, at two wavelengths for various porosity values. The mixing rule of Bruggeman (Pl refer Voshchinnikov et al. 2007) was used to calculate (m',k')

\begin{tabular}{lccr}
\hline$\lambda(\mu \mathrm{m})$ & porosity $f \%$ & $m^{\prime}$ & $k^{\prime}$ \\
\hline 0.485 & 0 & 1.692 & 0.0492 \\
0.485 & 10 & 1.621 & 0.0442 \\
0.485 & 20 & 1.549 & 0.0391 \\
0.485 & 30 & 1.477 & 0.0338 \\
0.485 & 40 & 1.404 & 0.0283 \\
0.485 & 50 & 1.331 & 0.0228 \\
0.684 & 0 & 1.672 & 0.0185 \\
0.684 & 10 & 1.603 & 0.0166 \\
0.684 & 20 & 1.533 & 0.0147 \\
0.684 & 30 & 1.463 & 0.0127 \\
0.684 & 40 & 1.392 & 0.0107 \\
0.684 & 50 & 1.322 & 0.0086 \\
\hline
\end{tabular}

The main aim of the present work is not to exactly reproduce the observed cometary data, but to discuss on the effect of dust particle porosity on scattered light ( with a realistic dust particle model), which can be useful for cometary studies. Matching our calculated polarization and color values with the observed ones will require more computational time, to fine tune the free parameters like porosity, power-law index and pyroxene to organic ratio etc. as discussed above. This we postpone for future work.

\section{Conclusions}

The aim and main emphasis of the present work, was to develop a new generic algorithm able to generate realistic porous aggregates with tunable porosity and tunable dust particle size distribution. We focused on porous spheres, but generalization of the process to other shapes (e.g. porous ellipsoids) is straightforward.

Using the DDSCAT light scattering code, we showed that such porous dust particles can generate meaningful polarization values for comets, and hence provide a tool to study in a systematic way the effect of dust particle porosity on cometary light scattering phenomenon, such as polarization, color, etc.

From the analysis of the simulated polarization curves, we find that with the increase in porosity (from $0 \%$ to $50 \%$ ) both the maximum and minimum polarization values change in a non-monotonic way. Indeed, the negative polarization branch becomes deeper with the increase in porosity and then again it becomes shallower. This phenomenon is true for both the wavelengths $\lambda=0.485 \mu \mathrm{m}$ and $=0.684 \mu \mathrm{m}$ and with the three values of power law index $\alpha=1.8,2.8$ and 3.8 considered here. 
More specifically, we have successfully generated polarization values which match grossly with the observed polarization data for comet Halley. In the first step, this was done first for porous pyroxene, to study the effect of porosity on polarization and compare with Effective Medium Theory (EMT).

It has been found that, our model of connected dipoles does not produce appreciably different values of polarization as compared to Effective Medium Theory (EMT).

Then, taking a more realistic composite model (pyroxene +organic), we make comparison with the observed cometary polarization. It appears that the matching with the observed polarization data of comet Halley (at wavelength $0.485 \mu \mathrm{m}$ ) is better with $f=40 \%$ porosity and $\alpha=2.8$ size distribution power law index, though we should adjust the pyroxene to organic ratio to get a better fit.

A sequel work is planned to find the optimum values of the two parameters $f, \alpha$ and also pyroxene to organic ratio for the composite model which realize the least square fitting to the observed data.

On the other hand, the simulated cometary color values show only weak dependence on the porosity values or on the scattering angles with the noticeable exception of the domain around the back-scattering direction. At the porosity $f=40 \%$ and power law index $\alpha=2.8$, the simulated color value $C \simeq-0.6$ ( for pyroxene and composite model) does not seem to match well with the average observed color value reported earlier by Kolokolova et al. (1997) which is $\simeq-0.024$. This discrepancy may come from the underestimation of the upper limit of the dust particle radius or from the size dependence of single dust particle albedo.

Generally, the simulated color values solely appear to be dependent on the power law index of dust particle size distribution function. Thus the dust color depends on dust particle size and this finding confirms a trend reported by Kolokolova et al. (1997) earlier.

We have also calculated the color slope values for the two dust particle models, as this parameter is often referred in literature.

At last, we showed that the Effective Medium Theory can only be used qualitatively to understand various features in the polarization or color behaviors. If quantitative achievement is required, more detailed theory has to be used, such as the DDA method applied to porous particles as generated by the algorithm given in the present paper.

\section{Acknowledgements}

R.B. thanks the Assam University, Silchar, for hospitality in the framework of 'Global Initiative for Academic Network' (GIAN) programme from MHRD, April 2016. The authors AKS and RV thank Erasmus Mundus - NAMASTE programme for funds to do 
this collaborative work. Finally, we are thankful to anonymous referees of this paper, due to whose comments we believe the quality of paper has improved.

\section{References}

[1] vom Humboldt A., Otte E.C., 1997, John Hopkins University Press

[2] Hall J.S., 1949, Science, 109(2825), 166-167

[3] Hiltner W., 1949, Science, 109, 165

[4] Greenberg, J.M., Hage, G., 1990,Astrophys J, 361, 260-74

[5] Lord Rayleigh. 1871, Philosophical Magazine,41, p.107-120

[6] Maxwell J.C. 1873 Nature VIII, 437-441

[7] van de Hulst H.C., Light scattering by small particles, Wiley, New York, 1957

[8] Mishchenko, M. I., \& Travis, L. D. 1994, Opt. Comm., 109, 16

[9] Draine B.T. in: Mishchenko M.I., Hovenier, J.W., Travis, L.D., editors. Light Scattering by Nonspherical Particles, Theory, Measurements, and Applications. New York: Academic Press, 2000. p. 131-145

[10] Taflove A., Hagness S.C., Computational Electrodynamics: the Finite-Difference Time-Domain Method, 2nd ed. Norwood: Artech House, 2005

[11] Jullien, R., Botet, R. 1987, Aggregation and Fractal Aggregates, World Scientific Publishing, Singapore

[12] Shen Y., Draine B. T., Johnson E. T., 2008, ApJ, 689, 260

[13] Shen Y., Draine B. T., Johnson E. T., 2009, ApJ, 696, 2126

[14] Brownlee D. E., 1985, Ann. Rev. Earth Planet. Sci., 13, 147

[15] Das H.S., D. Paul, A. Suklabaidya and A. K. Sen,2011, MNRAS 416,94-100

[16] Horz, F., Bastien R, Borg J, Bradley JP, Bridges JC, Brownlee DE, Science, 2006, 314(5806)

[17] Bentley MS, Schmied R, Mannel T, Torkar K, jeszenszky H, Romstedt J, Nature, 2016, 537(7618), 73-5.

[18] Muinonen K, Nousuiainen T, Fast P, Lumme K, peltoniemi J, JQSRT, 1996, 55(5), 577-601.

[19] Xing Z., Hanner M. S., 1997, A\&A, 324, 805

[20] Kimura H., 2001, J. Quant. Spectrosc. Radiat. Transfer, 70, 581 
[21] Kimura H., Kolokolova L., Mann I., 2006, A\&A, 449, 1243

[22] Wurm G., Blum J., 1998, Icarus, 132, 125

[23] Gustafson B.S., Kolokolova L., 1999, J. Geophys. Res., 104, 31711

[24] Hadamcik,E., Sen,A.K., Levasseur-Regourd,A.C., Gupta,R., Lasue,J., 2010.Astron.Astrophys.517,A86.

[25] Hadamcik,E., Sen,A.K., Levasseur-Regourd,A.C., Gupta,R., Lasue,J., Botet,R.,2013, Icarus222,774-785.

[26] Hadamcik,E., Sen,A.K., Levasseur-Regourd,A.C., RoyChoudhury,S., Lasue,J., Gupta, R. ,2014. Meteor.Planet.Sci.49(1),36-44.

[27] Petrova E. V., Tishkovets V. P., Jockers K., 2004, Sol. Syst. Res., 38, 309

[28] Tishkovets V. P., Petrova E.V., Jockers K., 2004, J. Quant. Spectrosc. Radiat. Transfer, 86, 241

[29] Gupta R., Vaidya D. B., Bobbie J.S; Chylek Petr, Astrophysics \& Space Science, 301 , issue 1, pp. 21-31, 2006

[30] Bertini I., Thomas N., Barberi C., 2007, A\&A, 461, 351

[31] Kolokolova L., Kimura H., Kiselev N., Rosenbush V., 2007, A\&A, 463,1189

[32] Moreno, F. , Muoz, O. , Guirado, D. and Vilaplana, R., 2007, JQSRT, 106, 348

[33] Das H.S., Sen A. K., 2011, J. Quant. Spectrosc. Radiative Transfer, 112, 1833

[34] Jiang, S.Z., editor. Focus on Combustion Research. New York: Nova Science Publishers, 2006

[35] Barabási A.L., Vicsek T., 1990, J. Phys.A, 23, L729

[36] Meakin P., 1983, J. Colloid Interface Sci., 96, 415

[37] Meakin P., 1984, Phys. Rev. A, 29, 997

[38] Vilaplanaa R., Moreno F., Molina A., 2006, JQSRT,100,415

[39] Vilaplana R., Luna R., Guirado, D., 2011, JQSRT,112, 1838

[40] Demers A., Greene D., Hauser C., Irish W., Larson J.,Shenker S., Sturgis H., Swinehart D., Terry D. in Proceedings of the 6th Annual ACM Symposium on Principles of Distributes Computing (PODC' 87), 1987. p. 1-12

[41] Sen A .K, Deshpande M R, Joshi U C, Rao N K, Raveendran A V, 1991, Astron \& Astrophys, 242, 496

[42] Rotundi A., and other 39 authors., 2015, Science,347,6220, p.3905-1 
[43] Kolokolova L, Jockers K, Chernova G 1997, ICARUS 126, 351-361

[44] Kolokolova L, Jockeres K, Gustafson Bo A S, and Gunther L, JOURNAL OF GEOPHYSICAL RESEARCH, 2001, VOL. 106, NO. E5, 10,113-10,127

[45] Mukai T, Mukai S, \& Kikuchi S, 1987, Astron. Astrophys., 187, 650

[46] Zubko E.,Earth, Planets Space, 65(3),13948 .

[47] Bohren, C. F., Hofman, D. R., 1983. Absorption and Scattering of Light by Small Particles. Wiley, New York.

[48] Draine B.T., Flatau P.J. Discrete-dipole approximation for scattering calculations. J Opt Soc Am A 1994;11:1491-1499.

[49] Mishchenko MI, Hovenier JW , Travis LD .Academic Press San Diego, 2000.

[50] Seizinger A., Kley W., 2013, Astron \& Astrophys, A65

[51] Wang C. Y., Liu P. L., Bassingthwaighte J. B., 1995, J. Phys.A Math. Gen., 28, 2141

[52] Lumme K , Rahola J., 1994, Astrophys J, 425, 653-67.

[53] Wolff MJ , Clayton GC, Martin PG , Schulte-Ladbeck RE., 1994, Astrophys $\mathrm{J}, \mathrm{b} 423,41225$.

[54] Wolff MJ , Clayton GC, Gibson SJ., 1998, Astrophys J, 503(2), 815.

[55] Maxwell-Garnett J.C.1904 Philos Trans R Soc A, 203: 385-420.

[56] Zubko E, Petrov D, Shkuratov Y, Videen G., 2005, Appl Opt,44(30),647985 .

[57] Zubko E , Shkuratov Y , Mishchenko M , Videen G., 2008, JQSRT,109(12),2195206.

[58] Zubko E, Shkuratov Y, Videen G., 2015, JQSRT,150, 4254.

[59] Jenniskens P., 1993, Astron Astrophys, 274, 653.

[60] Hanner M.S., Bradley J.P. Composition and mineralogy of cometary dust, 2004.

[61] Ahearn M, Schleicher D , Millis R , Feldman P, Thompson D., 1984, Astron J, $89,57991$.

[62] Mishchenko MI , Dlugach ZM , Zakharova NT., 2014, Opt Lett, 39(13), 39358.

[63] Mishchenko MI, Dlugach JM , Liu L., 2016A, JQSRT, 178, 28494.

[64] Mishchenko MI , Dlugach JM , Yurkin MA , Bi L , Cairns B , Liu L , et al., 2016b, Phys Rep, 632, 175.

[65] Voshchinnikov N.V., Videen G., and Henning T., 2007 Applied Optic, vol 46, Issue 19, 4065 
[66] Vaidya D.B, Gupta R, Snow T.P, 2007 MNRAS 379, 791-800

[67] Lasue J , Levasseur-Regourd AC , Hadamcik E, Alcouffe G.,2009, Icarus, 199(1), 12944 